\title{
Resurfacing the donor sites of reverse sural artery flaps using thoracodorsal artery perforator flaps
}

\author{
Se Won Oh, Seong Oh Park, Youn Hwan Kim \\ Department of Plastic and Reconstructive Surgery, Hanyang University College of Medicine, Seoul, Korea
}

\begin{abstract}
Background The reverse sural artery (RSA) flap is widely used for lower extremity reconstruction. However, patients sometimes suffer from donor site complications such as scar contracture and paresthesia, resulting in dissatisfaction with the aesthetic outcomes. This study investigated the characteristics of donor site morbidity associated with RSA flaps and described our experiences of dealing with complications by performing resurfacing surgery using thoracodorsal artery perforator (TDAP) flaps.
\end{abstract}

Methods From April 2008 to August 2018, a total of 11 patients underwent contracture release and resurfacing surgery using TDAP flaps due to donor morbidity associated with RSA flaps. All affected donor sites were covered with a skin graft, the most common of which was a meshed split-thickness skin graft (six cases).

Results Eight of the 11 patients (72.7\%) suffered from pain and discomfort due to scar contracture, and seven (63.6\%) complained of a depression scar. The donor sites were located $6.3 \pm 4.1 \mathrm{~cm}$ below the knee joint, and their average size was $140.1 \mathrm{~cm}^{2}$. After resurfacing using TDAP flaps, significant improvements were found in the Lower Extremity Functional Scale (LEFS) scores and the active and passive ranges of motion (AROM and PROM) of the knee joint. The LEFS scores increased from 45.1 to 56.7 postoperatively $(P=0.003)$, AROM increased from $108.2^{\circ}$ to $118.6^{\circ}(P=0.003)$, and PROM from $121.4^{\circ}$ to $126.4^{\circ}(P=0.021)$.

Conclusions Planning of RSA flaps should take into account donor site morbidity. If complications occur at the donor site, resurfacing surgery using TDAP flaps achieves aesthetic and functional improvements.

Keywords Limb salvage / Lower extremity / Reverse sural artery flap / Donor site morbidity / Thoracodorsal artery perforator flap

\author{
Correspondence: Youn Hwan Kim \\ Department of Plastic and \\ Reconstructive Surgery, Hanyang \\ University College of Medicine, 222-1 \\ Wangsimni-ro, Seongdong-gu, Seoul \\ 04763 , Korea \\ Tel: $+82-2-2290-8560$ \\ Fax: +82-2-2295-7671 \\ E-mail:younhwank@daum.net
}

Received: June 18, $2021 \bullet$ Revised: August 30, $2021 \bullet$ Accepted: September 14, 2021

pISSN: 2234-6163 • elSSN: 2234-6171 • https://doi.org/10.5999/aps.2021.01088• Arch Plast Surg 2021;48:691-698

\section{INTRODUCTION}

The reverse sural artery (RSA) flap is a fasciocutaneous or adipofascial flap that has been widely used for reconstruction of the distal lower extremity since its introduction in the 1980s [1-6]. Since the flap is thin and pliable, with a reliable blood supply, it is particularly useful for reconstruction of the distal tibial region, ankle, and foot. Moreover, microvascular surgery is not required. Due to its simplicity, it has gained popularity, and various technical modifications and harvesting methods have been developed to increase flap viability [7-10]. However, the flap can cause various problems. There is a risk of vascular complications and flap necrosis. Furthermore, some patients suffer from donor site morbidity and sural nerve injury caused by flap harvest. 
Most previous studies of RSA flaps have focused on surgical modifications to reduce flap congestion and partial loss, but donor site morbidity has received relatively little attention.

To secure abundant vascularity of the RSA flap, the adipofascial pedicle should be of sufficient width and length to include the gastrocnemius fascia. If primary closure is not possible due to the large size of the donor site, a graft is required to cover the gastrocnemius muscle. Some patients who have received splitthickness skin grafts (STSGs) for donor site coverage have complained of scar contracture, dysesthesia, and aesthetic problems $[7,11,12]$. Furthermore, because the RSA flap uses the vascular axis around the sural nerve as a pedicle, the sural nerve is sacrificed during flap elevation, leading to paresthesia [13].

Recent studies have investigated morbidity at the donor site and the complications caused by flap harvest. To reduce the size of the donor site and prevent morbidity, Chi et al. [14] introduced a modified flap with a narrow pedicle and presented a method for donor site coverage with a local relaying island flap based on a perforator without an STSG. Despite these efforts, skin grafts are inevitably needed to cover large donor sites. Therefore, patients still suffer from donor site complications and require attention because of donor site morbidity. The purpose of this study was to investigate the characteristics of donor site morbidity associated with RSA flaps and to describe our experience of dealing with these complications by performing resurfacing surgery using thoracodorsal artery perforator (TDAP) flaps.

\section{METHODS}

\section{Study design}

This investigation was approved by our Institutional Review Board (IRB No. 2021-02-013). The present study is a retrospective analysis of 11 patients who underwent resurfacing surgery using TDAP flaps for complications of the donor sites of RSA flaps between April 2008 and August 2018. Of the 11 patients, six were women $(55.5 \%)$, and their mean age was 44.3 years (range, 22-65 years). The average body mass index was $23.9 \pm 3.2 \mathrm{~kg} / \mathrm{m}^{2}$. Two of the patients had diabetes mellitus, one had hypertension, and nine had no underlying disease (Table 1). All the patients had undergone RSA flap surgery at other institutions for traumatic crush injuries, and the donor site defects had been covered with skin grafts. The patients complained of functional problems during ambulation and pain caused by scar contracture at the donor site, as well as aesthetic dissatisfaction due to the depression scars.

The affected donor sites were classified according to the type of skin graft used for donor site coverage. To estimate the location of each donor site, the distance from its upper margin to the
Table 1. Demographic characteristics of patients

\begin{tabular}{lc}
\hline Variable & Value \\
\hline No. of patients & 11 \\
Male & $5(45.5)$ \\
Female & $6(55.5)$ \\
Age $(\mathrm{yr})$ & $44.3 \pm 14.8$ \\
Body mass index $\left(\mathrm{kg} / \mathrm{m}^{2}\right)$ & $23.9 \pm 3.2$ \\
Comorbidities & \\
Diabetes mellitus & $2(18.2)$ \\
Hypertension & $1(9.1)$ \\
No underlying disease & $9(81.8)$ \\
\hline
\end{tabular}

Values are presented as number (\%) or mean \pm SD.

knee joint was determined, and its size, including the surrounding scar contracture skin and tissue, was measured.

To evaluate function, the active and passive ranges of motion (AROM and PROM) of the knee joint were measured as the difference between the angle of flexion and extension of the knee, using a goniometer. The Lower Extremity Functional Scale (LEFS) was also scored preoperatively and at a 1-year follow-up. The LEFS is a questionnaire evaluating the functional status of the lower extremities. It consists of a total of 20 items, each of which is assigned a score from 0 (extreme difficulty/unable to perform activity) to 4 (no difficulty). A perfect score of 80 indicates no functional limitation $[15,16]$.

The Wilcoxon signed-rank test was used to analyze range of motion (ROM) and LEFS scores. The statistical analysis was performed with SAS version 9.4 (SAS Institute Inc., Cary, NC, USA), and a P-value $<0.05$ was considered to indicate statistical significance.

\section{Surgical technique}

Due to the possibility of tissue or vessel injury resulting from the crush injury of the lower extremity, computed tomography (CT) angiography was used to select appropriate recipient vessels for resurfacing surgery using the TDAP flap and to evaluate the scar tissue status preoperatively. We performed wide excision on the affected donor site of the RSA flap including the surrounding scar tissue. During the dissection, if an adhesion or pathologic fibrotic tissue was encountered, the contracture was released by adhesiolysis and removal of the scar tissue. Since the defect resulting from contracture release and excision of scar tissue is larger than the donor area, the TDAP flap has to be large enough to cover the defect. The harvested TDAP flaps were shaped and thinned according to the contours and depths of the defects.

A reliable artery with sufficient blood flow and patency on CT angiography was selected as the recipient artery, and an accompanying vein was used as the recipient vein. The anterior tibial 
artery (ATA), posterior tibial artery (PTA), or inferior medial geniculate artery was selected, depending on which was most suitable. The length of the TDAP pedicle should be appropriate for the location and depth of the target recipient vessel. Defects after wide excision of the donor site of the RSA flap should be sufficiently undermined to prevent tension. After the flap was inset into the defect, target recipient vessels were identified and micro-anastomosis was performed.

A daily dose of $10 \mu \mathrm{g}$ of prostaglandin E1 (Eglandin; Mitsubishi, Osaka, Japan) was administered intravenously as a vasodilator for 1 week postoperatively. In patients with arterial insufficiency or thrombotic tendency, $800 \mathrm{IU} / \mathrm{hr}$ of heparin was administered intravenously over the first 6 postoperative hours, and $400 \mathrm{IU} / \mathrm{hr}$ thereafter for 5 days.

\section{RESULTS}

Of the 11 patients, eight (72.7\%) suffered from pain and discomfort due to scar contracture, and seven (63.6\%) complained of a depression scar. Several patients also complained of dysesthesia, three of a tingling sensation, and two of numbness. The target regions of the RSA flap were the hindfoot in five patients (45.6\%), the middle one-third of the tibia in five patients (45.6\%), and the forefoot in one patient. The donor site was covered with a simple STSG in three cases $(27.3 \%)$ and a meshed STSG in six cases (54.5\%). In the other two cases, artificial dermis was used on the wound bed, which was covered with a meshed STSG. The mean size of the affected donor sites, including the surrounding scar tissue, was $140.1 \mathrm{~cm}^{2}$ (95\% confidence interval, $86.7-193.0 \mathrm{~cm}^{2}$ ), and the sites were located $6.3 \pm 4.1 \mathrm{~cm}$ below the knee joint. The mean time from RSA flap surgery to donor site resurfacing was 35.2 months (range, 8-120 months) (Table 2).

TDAP flaps were used for resurfacing in all 11 cases, and in one case, an anterolateral thigh flap was used at the same time to cover additional wounds around the donor site. The mean length of the flap pedicle vessel was $9.8 \pm 1.4 \mathrm{~cm}$. The PTA was used as the recipient vessel in seven cases (63.6\%), the ATA in two cases, and the inferior medial geniculate artery in two cases. The mean follow-up period was 28 months (range, 12-60 months) (Table 3).

After resurfacing surgery, lower extremity function improved and the ROM of the knee joints increased. The LEFS score increased from 45.1 preoperatively to 56.7 postoperatively $(\mathrm{P}=$ $0.003)$. The AROM of the knee joint rose from $108.2^{\circ} \pm 7.2^{\circ}$ to $118.6^{\circ} \pm 8.4^{\circ}(\mathrm{P}=0.003)$, and the PROM increased from $121.4^{\circ} \pm 9.2^{\circ}$ to $126.4^{\circ} \pm 7.1^{\circ}(\mathrm{P}=0.021)$. All patients experienced reduced pain and discomfort of the lower extremities and
Table 2. Characteristics of RSA flaps with complications at the donor site

\begin{tabular}{lc}
\hline Variable & Value $(\mathrm{n}=11)$ \\
\hline Chief complaint & \\
Scar contracture & $8(72.7)$ \\
Depression scar & $7(63.6)$ \\
Tingling sensation & $3(27.3)$ \\
$\quad$ Numbness & $2(18.2)$ \\
Operation side & \\
$\quad$ Left & $5(45.5)$ \\
$\quad$ Right & $6(55.5)$ \\
Target region of the RSA flap & \\
$\quad$ Hindfoot & $5(45.6)$ \\
$\quad$ Middle one-third of tibia & $5(45.6)$ \\
$\quad$ Forefoot & $1(9.1)$ \\
Previous skin graft for the donor site of the RSA flap & \\
$\quad$ STSG & $3(27.3)$ \\
Meshed STSG & $6(54.5)$ \\
Artificial dermis + meshed STSG & $2(18.2)$ \\
Distance from knee joint (cm), mean \pm SD) & $6.3 \pm 4.1$ \\
Size of the donor site with complications (cm²), mean & $140.1(86.7-193.0)$ \\
$\quad$ (95\% Cl) & \\
Period from the RSA flap to resurfacing operation (mo), & $35.2(8-120)$ \\
mean (range) & \\
\hline
\end{tabular}

Values are presented as number (\%) unless otherwise indicated.

RSA flap, reverse sural artery flap; STSG, split-thickness skin graft; Cl, confidence interval.

a) The distance from the popliteal crease to the upper margin of the defect was measured.

Table 3. Operative variables for resurfacing surgery

\begin{tabular}{lc}
\hline Variable & Value \\
\hline Flap composition of resurfacing donor site & \\
TDAP & $10(90.9)$ \\
TDAP + ALT & $1(9.1)$ \\
Flap recipient vessela) & \\
ATA & $2(18.2)$ \\
PTA & $7(63.6)$ \\
Inferior medial geniculate artery & $2(18.2)$ \\
Length of flap pedicle vessel (cm), mean \pm SD & $9.8 \pm 1.4$ \\
Follow-up period (mo), mean (range) & $28(12-60)$
\end{tabular}

Values are presented as number (\%) unless otherwise indicated.

TDAP, thoracodorsal artery perforator; ALT, anterolateral thigh; ATA, anterior tibial artery; PTA, posterior tibial artery.

a)The accompanying vein was used as the recipient vein for anastomosis

were satisfied functionally and aesthetically.

All the TDAP flaps survived, although there was partial loss of the distal flap in one case. Delayed wound healing was found at the donor site of the TDAP flap in two cases, but there were no other donor site complications (Table 4).

\section{Case 1}

A 56-year-old woman suffered a crush injury of the left foot in a traffic accident involving a bus and underwent forefoot reconstruction using an RSA flap. The donor site of the RSA flap was 
covered with a meshed STSG. The patient suffered from pain and discomfort during ambulation due to an unstable scar on the forefoot and scar contracture at the donor site of the RSA flap, and complained of a tingling sensation. She also complained of aesthetic dissatisfaction with the depression scar.

Nineteen months after insertion of the RSA flap, a two-stage operation was planned to resurface the left forefoot and donor site using bilateral TDAP flaps. The unstable scar on the forefoot was removed and reconstructed using the right-side TDAP

Table 4. Outcome-related variables of resurfacing surgery

\begin{tabular}{lccc}
\hline Variable & Preoperative & Postoperative & P-value \\
\hline AROM of knee joint $\left({ }^{\circ}\right)^{a)}$ & $108.2 \pm 7.2$ & $118.6 \pm 8.4$ & 0.003 \\
PROM of knee joint $\left({ }^{(}\right)^{a)}$ & $121.4 \pm 9.2$ & $126.4 \pm 7.1$ & 0.021 \\
LEFS $^{\text {b) }}$ & $45.1(35.7-54.5)$ & $56.7(47.4-66.1)$ & 0.003 \\
Postoperative complications & & \\
$\quad$ TDAP flap & & \\
$\quad$ Flap partial loss & $1(9.1)$ & \\
$\quad$ None & $10(90.9)$ & \\
Donor site of TDAP flap & \\
$\quad$ Delayed wound healing (>3 wk) & $2(18.2)$ \\
$\quad$ None & $9(81.8)$ & \\
\hline
\end{tabular}

Values are presented as mean $\pm \mathrm{SD}$, mean $(95 \% \mathrm{Cl})$, or number (\%).

AROM, active range of motion; PROM, passive range of motion; LEFS, Lower Extremity Functional Scale; TDAP, thoracodorsal artery perforator; $\mathrm{Cl}$, confidence interval.

a) The range of motion was calculated by measuring the angle between flexion and extension of the knee joint; ${ }^{b}$ The total score is the sum of 200 items and is calculated as the highest score of 80 . flap in the first-stage operation. Two weeks later, a resurfacing operation using the left-side TDAP was performed at the donor site of the reverse sural flap as the second-stage operation. After removing the depression scar and performing contracture release around the knee joint, the defect measured about $14 \times 9$ $\mathrm{cm}$. The left-side TDAP flap was harvested and anastomosed to the PTA.

Both flaps survived without complications. The patient's pain and discomfort during ambulation were reduced. The preoperative LEFS score was 34 points, and the postoperative LEFS score measured one year later was 48 points, showing functional improvement. The patient was also satisfied with the aesthetic outcomes (Fig. 1).

\section{Case 2}

A 22-year-old man underwent reconstruction by transposition of a sural flap due to a defect in the anterior tibial region caused by a motorcycle accident. For coverage of the donor site of the RSA flap, a meshed STSG was used. The patient complained of pain and discomfort resulting from scar contracture deformity of the donor site. Twenty-six months later, reconstruction was performed using a TDAP flap to resurface the medial gastrocnemius and anterior tibial sites. The scar tissue was removed, and contracture release was performed by undermining the surrounding area. The TDAP flaps were harvested from the right side according to the shape and depth of the defect, which mea-
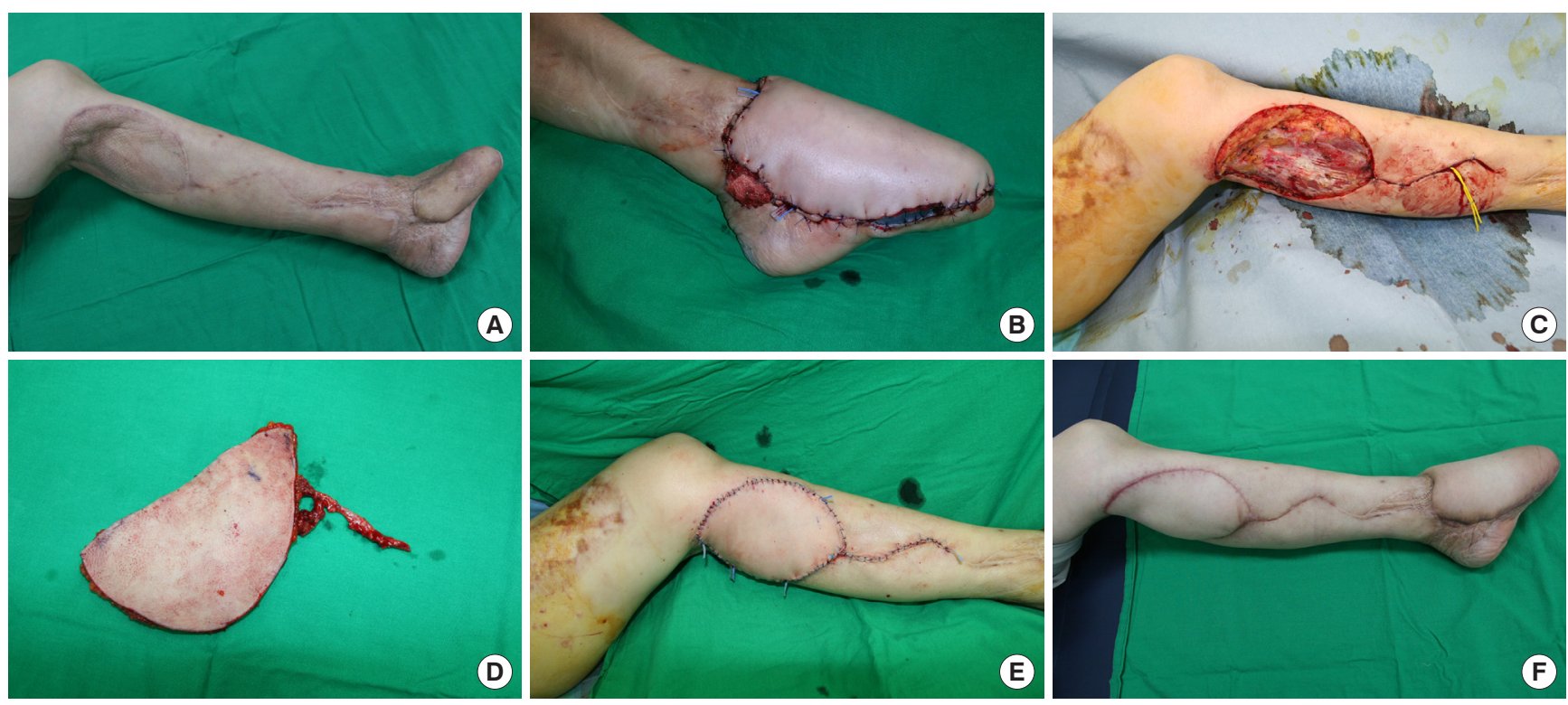

Fig. 1. Case 1. (A) Preoperative photography of forefoot reconstruction using reverse sural flaps with meshed split-thickness skin graft for coverage of the donor site. (B) In the first-stage operation, the forefoot was resurfaced using a right-side thoracodorsal artery perforator (TDAP) flap. (C) Intraoperative photograph of a $14 \times 9 \mathrm{~cm}^{2}$ defect in the second-stage operation after removing scar contracture tissue from the donor site with complications. (D) Intraoperative photograph of the harvested left-side TDAP flap. (E) After the flap was inset, anastomosis was performed using the posterior tibial artery as a recipient vessel. (F) Four months later, the flap survived without complications. 

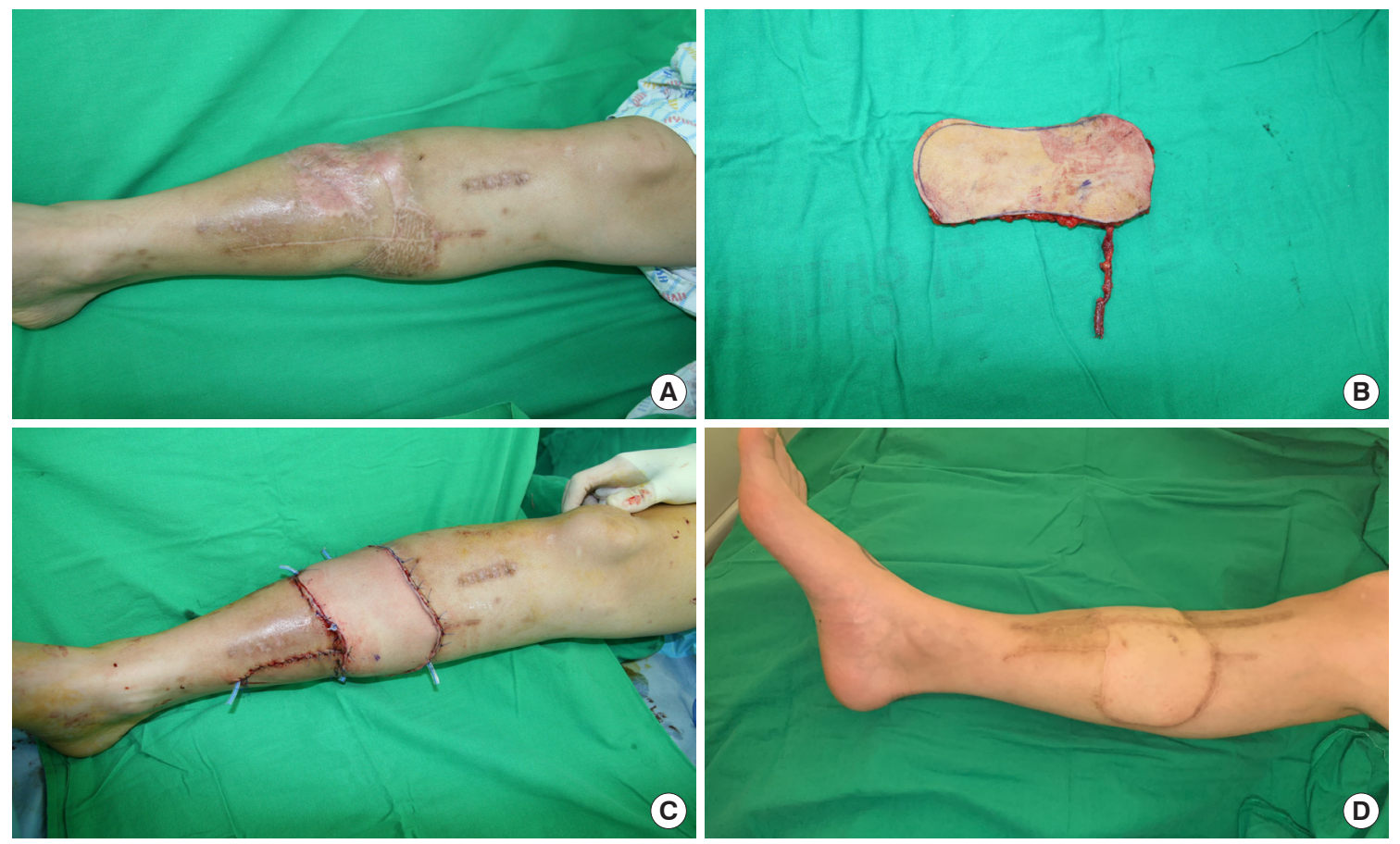

Fig. 2. Case 2. (A) A 22-year-old male underwent reconstruction by transposing sural flaps to defects in the anterior tibial area, and a meshed split-thickness skin graft was performed at the donor site. (B) Intraoperative photograph of an $18 \times 9 \mathrm{~cm}^{2}$ thin thoracodorsal artery perforator (TDAP) flap. (C) Postoperative photograph of resurfacing surgery using the thin TDAP flap. (D) Photograph after 2 years.

sured $18 \times 9 \mathrm{~cm}$. The flaps were thinned by removing adipose tissue due to the thinness of the tissue of the anterior tibial region. End-to-side anastomosis was performed on the thoracodorsal perforator and PTA.

Partial loss of the distal flap (about $1 \times 4 \mathrm{~cm}$ ) occurred postoperatively, but the problem was solved by an additional skin graft. Outpatient follow-up was performed for 26 months. The patient's discomfort in the lower extremity during daily life due to the scar contracture deformity was reduced. The preoperative LEFS score of 56 points increased to 70 points 1 year postoperatively, and the contours improved aesthetically (Fig. 2).

\section{DISCUSSION}

The RSA flap is a useful option for distal lower extremity reconstruction because it does not require microvascular surgery and has a reliable blood supply. However, donor site morbidity has not generally been taken into account. Skin grafts are usually considered necessary for defects at the donor site where primary closure is not possible [17]. The use of STSGs can lead to hypertrophic scars and inappropriate color or texture matching $[18,19]$. Yilmaz et al. [7] reported hypertrophic scars in two out of seven patients who had skin grafts at the donor site. In the case of meshed STSGs, the appearance of the surface in particular may be poor. Furthermore, due to their thinness, contour de- formity and irregularity occur in the coverage of deep defects. Because the donor site of the RSA flap is located below the knee, these wounds are readily visible, which increases patient dissatisfaction.

Our patients complained not only of aesthetic dissatisfaction, but also functional problems of the lower extremity. Most of them complained of pain and discomfort in the lower extremities caused by scar contracture, and they experienced functional problems in walking or daily life. Although the patients had lower preoperative LEFS scores than the healthy population due to their traumatic crush injuries of the lower extremities, contracture was released by resurfacing surgery, resulting in significant improvements in their LEFS scores [16]. Not all patients exhibited limited ROM in the knee joint. However, some experienced pain or discomfort during ambulation and complained of functional problems. ROM increased postoperatively as pain improved. Although they did not use RSA flaps, in a study of donor morbidity of the anterolateral thigh flap, Kimata et al. [20] reported that, after using STSGs to cover donor defects of the lower extremity, the ROM of the joint at the donor site was sometimes limited by pain during knee flexion due to adhesions between the skin graft and muscle. There were also tingling sensations and dysesthesia in the skin graft areas. In 2011, Kneser et al. [12] studied 24 patients and found that $58 \%$ of them had hypoesthesia in the skin graft region of the donor site, with an av- 
erage area of the hypoesthetic region of $204.9 \pm 127.2 \mathrm{~cm}^{2}$.

The donor sites of the RSA flaps were on average $6.3 \mathrm{~cm}$ below the knee joint. Scar contracture close to the knee joint may affect the motion of the knee joint and cause functional impairment [20]. The average size of the donor site was $140.1 \mathrm{~cm}^{2}$, and since primary closure was not possible in any of the patients, the donor sites were covered with STSGs. Meshed STSGs were the most common (54.5\%). Although meshed STSGs have the advantage of being able to cover a large area, they are known to induce more contracture deformity than STSGs [21]. In two cases, artificial dermis was used with the meshed STSGs. There are studies showing that the use of artificial dermis in the recipient bed of STSGs renders them resistant to contracture, but the problem was not completely solved in this study [22,23]. Furthermore, contracture release and resurfacing using artificial dermis with STSGs can be a valuable option for dealing with complications of the donor sites of RSA flaps. However, since our patients had large donor sites and severe adhesions between the skin grafts and muscles, we could not ignore the possible recurrence of contracture after resurfacing using skin grafts. At the same time, it was not possible to obtain sufficient tissue for local flaps. Therefore, in view of the severity of the symptoms and functional problems, we decided to plan resurfacing using free flaps.

At our institution, TDAP flaps are used for resurfacing surgery after scar tissue excision and for contracture release because of their multiple advantages. A sufficiently long and reliable donor vessel must be harvested; because the donor site of the RSA flap is near the knee joint, the surgical approach is inconvenient and a reliable recipient major vessel is located deep, so a long pedicle is required for microvascular surgery. Moreover, these flaps can be large enough to cover the donor site defect left by the RSA flap and they can be thinned appropriately to fit the depth of the recipient site and correct the contour deformity of the depression scar $[24,25]$. When considering the use of free flaps for resurfacing, it should be taken into account that donor site morbidity may occur. For instance, TDAP flaps could also cause donor site complications such as wound dehiscence and seroma, scar-related problems, and functional impairment of the shoulder. However, previous studies have suggested that the TDAP flap has low donor site morbidity and minimal dysfunction $[26,27]$. The use of TDAP flaps could reduce the donor site morbidity associated with resurfacing surgery.

This study has several limitations. Since all the patients underwent surgery with RSA flaps at other institutions, the outcomes of the RSA flaps and occurrence of complications may have varied according to the surgical techniques used. The adequacy of postoperative management could also have influenced the oc- currence of complications. However, we were not able to evaluate these factors. Additionally, this study involved only $11 \mathrm{pa}-$ tients and there was no control group that did not receive resurfacing surgery for donor site morbidity. The risk factors for donor site morbidity should be assessed in various patient groups, including those without donor site complications. Due to multiple trauma to the lower extremities resulting from crush injuries, patients' baseline LEFS values were lower than those of the healthy population. Most of our patients had injuries in the ankle region, making it difficult to evaluate the effect of the skin grafts on the ankle joints. If a skin graft over the gastrocnemius muscle is used for donor reconstruction, the ankle joint can be affected. These considerations should be taken into account when evaluating the characteristics of donor site morbidity and the outcomes of resurfacing surgery. Scar contracture at the donor site was not always associated with limited ROM of the knee joint. However, in some patients, ROM increased with postoperative pain relief. It will be important to demonstrate this connection objectively in further research. Aesthetics improved through the correction of contour deformities, and the patients were satisfied, but an objective evaluation was not performed.

Despite these limitations, this study did reveal the characteristics of donor morbidity resulting from creating RSA flaps, and provided experience of eliminating this morbidity by resurfacing surgery. To the best of our knowledge, no previous studies have analyzed the characteristics of donor site morbidity associated with RSA flaps. The patients in the present study had large donor sites close to the knee joint, and the donor defects had been reconstructed using skin grafts. Previous studies have suggested that the morbidity of donor sites in the lower extremity increases with donor flap size and proximity to the knee joint, and that larger skin grafts are more susceptible to contracture $[20,28,29]$. These findings suggest several ways to prevent donor site morbidity when planning RSA flaps. If the intended donor location is close to the knee joint, donor site complications need to be addressed. Furthermore, although STSGs are a good option for donor reconstruction, it is better in terms of scar contracture and aesthetic problems to perform primary closure or use local flaps where possible. For small to moderate-sized donor site defects, primary suture is recommended. If primary closure is not feasible, suture techniques such as Z-plasty can be used after sufficient undermining. For moderate-sized to large donor defects, one can reduce the donor area using tightening suture techniques and reconstruct the donor site with an island or local flap [28]. If there is an extensive crush injury of the lower extremity or there is insufficient tissue for an effective local flap, coverage with a skin graft should be considered. The pres- 
ence of the artificial dermis in the recipient beds of STSGs should make it possible to reduce the contracture and aesthetic problems that may occur with other types of skin grafts [21,29].

In conclusion, the RSA flap is a valuable reconstructive option in the distal lower extremities. However, donor site morbidity should be considered when planning reconstruction using the RSA flap. If complications occur with STSGs at the donor site, resurfacing surgery using TDAP flaps overcomes donor site morbidity and achieves aesthetic and functional improvements.

\section{NOTES}

\section{Conflict of interest}

Youn Hwan Kim is an editorial board member of the journal but was not involved in the peer reviewer selection, evaluation, or decision process of this article. No other potential conflicts of interest relevant to this article were reported.

\section{Ethical approval}

The study was approved by the Institutional Review Board of Hanyang University Hospital (IRB No. 2021-02-013) and performed in accordance with the principles of the Declaration of Helsinki. Written informed consent was obtained.

\section{Patient consent}

The patients provided written informed consent for the publication and the use of their images.

\section{Author contribution}

Conceptualization: SW Oh, SO Park, YH Kim. Data curation: SW Oh, YH Kim. Formal analysis: SW Oh, SO Park, YH Kim. Investigation: SW Oh, YH Kim. Methodology: SW Oh, SO Park, YH Kim. Project administration: SW Oh, SO Park, YH Kim. Resources: SW Oh, SO Park, YH Kim. Software: SW Oh, SO Park. Supervision: YH Kim. Validation: SW Oh, SO Park, YH Kim. Visualization: SW Oh, YH Kim. Writing - original draft: SW Oh, SO Park, YH Kim. Writing - review \& editing: SW Oh, SO Park, YH Kim.

\section{ORCID}

Se Won Oh

https://orcid.org/0000-0002-5358-085X

Seong Oh Park

https://orcid.org/0000-0001-8990-0635

Youn Hwan Kim

\section{REFERENCES}

1. Donski PK, Fogdestam I. Distally based fasciocutaneous flap from the sural region: a preliminary report. Scand J Plast

Reconstr Surg 1983;17:191-6.

2. Wee JT. Reconstruction of the lower leg and foot with the reverse-pedicled anterior tibial flap: preliminary report of a new fasciocutaneous flap. Br J Plast Surg 1986;39:327-37.

3. Hasegawa M, Torii S, Katoh H, et al. The distally based superficial sural artery flap. Plast Reconstr Surg 1994;93:101220.

4. Jeng SF, Wei FC. Distally based sural island flap for foot and ankle reconstruction. Plast Reconstr Surg 1997;99:744-50.

5. Ciofu RN, Zamfirescu DG, Popescu SA, et al. Reverse sural flap for ankle and heel soft tissues reconstruction. J Med Life 2017;10:94-8.

6. AlMugaren FM, Pak CJ, Suh HP, et al. Best local flaps for lower extremity reconstruction. Plast Reconstr Surg Glob Open 2020;8:e2774.

7. Yilmaz M, Karatas O, Barutcu A. The distally based superficial sural artery island flap: clinical experiences and modifications. Plast Reconstr Surg 1998;102:2358-67.

8. Al-Qattan MM. A modified technique for harvesting the reverse sural artery flap from the upper part of the leg: inclusion of a gastrocnemius muscle "cuff" around the sural pedicle. Ann Plast Surg 2001;47:269-78.

9. Buluc L, Tosun B, Sen C, et al. A modified technique for transposition of the reverse sural artery flap. Plast Reconstr Surg 2006; 117:2488-92.

10. de Rezende MR, Saito M, Paulos RG, et al. Reduction of morbidity with a reverse-flow sural flap: a two-stage technique. J Foot Ankle Surg 2018;57:821-5.

11. Baumeister SP, Spierer R, Erdmann D, et al. A realistic complication analysis of 70 sural artery flaps in a multimorbid patient group. Plast Reconstr Surg 2003;112:129-42.

12. Kneser U, Brockmann S, Leffler M, et al. Comparison between distally based peroneus brevis and sural flaps for reconstruction of foot, ankle and distal lower leg: an analysis of donor-site morbidity and clinical outcome. J Plast Reconstr Aesthet Surg 2011;64:656-62.

13. Kim H, Hu J, Chang H, et al. Sural nerve splitting in reverse sural artery perforator flap: anatomical study in 40 cadaver legs. Plast Reconstr Surg 2017;140:1024-32.

14. Chi Z, Chen Y, Chu T, et al. Distally based sural neuro-fasciocutaneous perforator flap for foot and ankle reconstruction: surgical modifications for flap pedicle and donor site closure without skin graft. J Plast Reconstr Aesthet Surg 2018;71:224-31.

15. Binkley JM, Stratford PW, Lott SA, et al. The Lower Extremity Functional Scale (LEFS): scale development, measurement properties, and clinical application. North American Orthopaedic Rehabilitation Research Network. Phys 
Ther 1999;79:371-83.

16. Dingemans SA, Kleipool SC, Mulders MA, et al. Normative data for the lower extremity functional scale (LEFS). Acta Orthop 2017;88:422-6.

17. Rudolph R, Ballantyne DL. Skin grafts. In: McCarthy JG, editor. Plastic surgery. Philadelphia: Saunders; 1990. p. 22174.

18. Hallock GG, Morris SF. Skin grafts and local flaps. Plast Reconstr Surg 2011;127:5e-22e.

19. Burnett LN, Carr E, Tapp D, et al. Patient experiences living with split thickness skin grafts. Burns 2014;40:1097-105.

20. Kimata Y, Uchiyama K, Ebihara S, et al. Anterolateral thigh flap donor-site complications and morbidity. Plast Reconstr Surg 2000;106:584-9.

21. Reid MJ, Currie LJ, James SE, et al. Effect of artificial dermal substitute, cultured keratinocytes and split thickness skin graft on wound contraction. Wound Repair Regen 2007;15: 889-96.

22. Min JH, Yun IS, Lew DH, et al. The use of Matriderm and autologous skin graft in the treatment of full thickness skin defects. Arch Plast Surg 2014;41:330-6.

23. Philandrianos C, Andrac-Meyer L, Mordon S, et al. Com- parison of five dermal substitutes in full-thickness skin wound healing in a porcine model. Burns 2012;38:820-9.

24. Lin CT, Huang JS, Yang KC, et al. Reliability of anatomical landmarks for skin perforators of the thoracodorsal artery perforator flap. Plast Reconstr Surg 2006;118:1376-86.

25. Hattori Y, Harima M, Yamashita S, et al. Superthin thoracodorsal artery perforator flap for the reconstruction of palmar burn contracture. Plast Reconstr Surg Glob Open 2020;8:e2695.

26. Lee KT, Mun GH. A systematic review of functional donorsite morbidity after latissimus dorsi muscle transfer. Plast Reconstr Surg 2014;134:303-14.

27. Lee KT, Kim A, Mun GH. Comprehensive analysis of donor-site morbidity following free thoracodorsal artery perforator flap harvest. Plast Reconstr Surg 2016;138:899-909.

28. Eser C, Gencel E, Kesiktas E, et al. A convenient flap for repairing the donor area of a distally based sural flap: gastrocnemius perforator island flap. J Plast Reconstr Aesthet Surg 2016;69:1109-15.

29. Hur GY, Seo DK, Lee JW. Contracture of skin graft in human burns: effect of artificial dermis. Burns 2014;40:1497503. 